\title{
A wide field fluorescence lifetime imaging system using a light sheet microscope
}

\author{
Phil M. Birch ${ }^{\mathrm{a}}$, Lamar Moore ${ }^{\mathrm{a}}$, Xiaofei Li ${ }^{\mathrm{b}}$, Roger Phillips ${ }^{\mathrm{b}}$, Rupert Young ${ }^{\mathrm{a}}$, and Chris \\ Chatwin ${ }^{\mathrm{a}}$ \\ ${ }^{a}$ Dept. of Engineering and Design, University of Sussex, Falmer, UK \\ ${ }^{\mathrm{b}}$ School of Life Sciences, University of Sussex, Falmer, UK
}

\begin{abstract}
Fluorescence lifetime imaging microscopy (FLIM) has allowed scientists to discern information about the chemical properties of biological processes and has become a vital tool in the life sciences and medical research communities. Measuring the spatial lifetime distribution of the fluorophores as well as the intensity distribution enables users to discern vital information about the chemical environment. It however, remains challenging and often involves slow scanning. We present a new microscope system based on light sheet illumination that uses a micro channel plate (MCP) device called a Capacitive Division Imaging Readout (CDIR) which has been developed by Photek Ltd. The device uses an array of capacitors to move the charge site from the MCP to four pre-amplifiers and time-over-threshold discriminators. This camera has the ability to image photons as well as measure the arrival time, enabling high speed FLIM imaging of biological samples.
\end{abstract}

Keywords: light sheet microscope, FLIM, MCP, photo multiplier tube

\section{INTRODUCTION}

Fluorescence Microscopy has become one of the driving forces behind much of our knowledge of the microscopic biological world in recent years. In particular, fluorescence lifetime imaging microscopy (FLIM) has allowed us to discern more information about the chemical properties of biological processes. However, conventional FLIM measures the life time with a point measurement and so requires scanning to produce a $2 \mathrm{D}$ image. The ability to measure the spatial 2D lifetime distribution of the fluorophores as well as the intensity distribution enables users to discern vital information about the chemical environment. It however, remains challenging. The possibility of photon damage to any organism under study means the illumination system must be limited in power. Therefore the emitted photon count from the subject for such microscopes is usually extremely limited, requiring a high degree of efficiency within the system, both optically and within any imaging sensor. In addition to this the timing resolution required to measure lifetimes are of the order of picoseconds.

Traditional methods to achieve two dimensional images involve scanning with a confocal or two photon microscope and collecting the photon timings with a point collector. This has the downside of being comparatively slow, taking of the order of a minute to produce two dimensional images. On top of this, pulsed laser systems capable of producing high powered, short pulse length, illumination suitable for two photon microscopy tend to be extremely expensive. To over come the speed issues, high speed, photon timing counters with spatial imaging capabilities therefore offer a desirable option to decrease measurement times.

An additional difficulty to overcome is the limited depth sectioning ability of conventional microscopes. To overcome this confocal or two photon methods have been demonstrated successfully in past. However, recently light sheet microscopy has been seen as a lower cost alternative.

One traditional method of measuring the arrival of a photon is via a photo-multiplier tube (PMT). PMTs consist of a photocathode for converting photons to photo-electrons, a dynode chain which amplifies the electron signal and finally a read-out anode used for collecting the amplified signal. The instrument response function (IRF) of PMTs are the characteristic which defines their timing resolution. This is closely related to the transit

Further author information: (Send correspondence to P.M.B.)

P.M.B.: E-mail: p.m.birch@sussex.ac.uk, Telephone: +44(0)1273 678553 
time spread (TTS) of the system which is the distribution in photon arrival times. ${ }^{1}$ The IRF/TTS determine the minimum time interval which can be accurately measured by the device. TTS for PMTs are usually on the order of picoseconds making them suitable for fluorescence lifetime measurements. Lower cost, solid state alternatives to PMTs exist in the form of single photon avalanche diodes (SPADs). ${ }^{2}$

Conventional PMTs and SPADs do not preserve any spatial information about an incoming photon. Methods like Confocal and Multi-photon microscopy make use of a scanning approach where the laser is scanned across the sample while the PMT/SPAD is treated as a single pixel device. Position information is from the scanning mechanism. Scanning methods generally tend to be slow and in some cases can be very damaging to the sample ${ }^{3}{ }^{4}$ Since low noise is required for good exponential fitting of the fluorescence lifetime decay, especially where more than one decay is present, this can mean that either high intensity sources are required or very long dwell times for each pixel. This exposure to intense light can be toxic to the sample and the long dwell times on each pixel means that the overall scanning process will be slow. Recent advances in microscope development have allowed for much faster scanning and reduced photo-bleaching/photo-toxicty, acquisition times for good photon statistics can still be below the desired rate for live cell imaging.

We have developed a custom solution to the problems mentioned above. In our system we side illuminated our sample with a sheet of excitation light produced by a pulsed 470nm laser diode. This light sheet offers the capability to optically section organisms without the expense of a two photon system. This type of microscope is known as a selective plane illumination microscope (SPIM) or as a light sheet microscope. ${ }^{5}$ The imaging path is then split into two paths: a high resolution EMCCD captures an intensity image allowing for alignment of the sample. The second path contains a multichannel plate (MCP) photomultiplier. MCP have a higher time resolution and an imaging capability when compared to conventional photo-multipliers. When a photon interacts with the MCP they are designed to localise the electron charge, making them suitable for imaging.

To read the charge, time and position from the MCP remains a challenge. Devices such as the H33D detector use a cross delay line anode $\mathrm{e}^{6,7}$ but with a relatively high cost. Other alternative systems exist such as SPAD arrays currently have a limited fill factor and low pixel count. We are demonstrating an alternative technology; a Capacitive Division Imaging Readout (CDIR) which has been developed by Photek Ltd. ${ }^{8}$ The device uses an array of capacitors to move the charge site from the MCP to four pre-amplifiers and time-over-threshold discriminators. This measures the timings of the charge with a timing jitter of only 30ps. From the four measurements, the position and the time of a photon can be determined.

This paper will describe the microscope. Section 2 will described the CDIR imaging system, as developed by Photek Ltd. The optical design and rest of the microscope will be discussed in section 3. The microscope has been calibrated using Oregon Green Bapta- 1 buffers with a varying degree of free $\mathrm{Ca}^{2+}$. The microscope shows a excellent degree of time resolution and temporal repeatability, this and our other results will be discussed in section 4 .

\section{SENSOR DESIGN}

The CDIR detector system was developed by Photek $^{8}$ and consists of a Photek Limited Resistive Sea MCP-PMT 240 (two MCPs), a CDIR anode, ${ }^{9}$ the NINO pre-amplifier/discriminator $\mathrm{ASIC}^{10}$ and the high performance timeto-digital (HPTDC) timing electronics. An incident photon is converted to a photo-electron by the photo-cathode and is the accelerated to the first MCP stage. The electron is amplified and the emerging electron charge cloud is localized by the resistive-sea anode which is made from a dielectric substrate. The charge is then capacitively transferred to the CDIR anode. The charge is distributed to the four corner anodes of the CDIR each of which are coupled to an input channel of the NINO ASIC. The NINO provides timing information on the start and duration of a charge pulse. The HPTDC then digitises the NINO output which is transferred to the PC via USB2.0 as 32-bit packets containing the timing information relative to the HPTDC frame trigger.

\subsection{CDIR Anode System}

The Capacitive Division Imaging Readout (CDIR) technique is a novel and conceptually simple approach to the problem of position resolved particle detection. ${ }^{9}$ The method is implemented using charge division (or sharing), similar in concept to modern touch-screen devices. The CDIR anode is comprised of a number of discrete charge 
sensitive pads, which are capacitively coupled to one another as shown in Figure 1. Charge is propagated through the detector surface via charge sharing until it is read out at each of the 4 corner anodes labelled A,B,C,D. The proportion of the charge which arrives at each channel is directly related to the input charge position and can be calculated using the following expressions:

$$
\begin{aligned}
& x=\frac{Q_{A}+Q_{B}}{Q_{A}+Q_{B}+Q_{C}+Q_{D}} \\
& y=\frac{Q_{A}+Q_{D}}{Q_{A}+Q_{B}+Q_{C}+Q_{D}}
\end{aligned}
$$

Where $Q_{i}$ is the charge collected at each anode, $i \in\{A, B, C, D\}$ and $(x, y)$ is the coordinate of the input charge.

Figure 2 shows how this couples to the whole detector stack. A photon strikes the photo-cathode plate, this charge is amplified by the MCP and couples with a resistive plate. This capacitively couples to the CDIR anode.

Due to the simplicity of its design and the ease with which the anode can be manufactured and coupled to other devices, the CDIR anode lends itself to a large cross section of imaging applications. The CDIR anode used in this set-up is forged on a PCB using discrete capacitive pads and with low-capacitance anode pins. This is an optimized design for the NINO ASIC which reduces the capacitive load on the electronics.

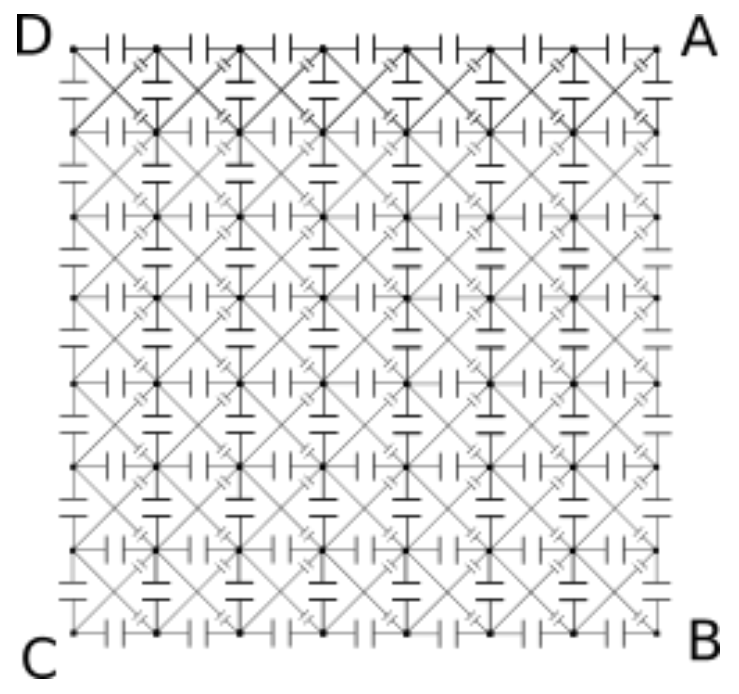

Figure 1. The capacitor layout of the CDIR detector. The capacitors allow for the transmission of the charge packets from the photon site to the four collection anodes: A, B, C and D

\subsection{NINO ASIC}

The NINO ASIC is a pre-amplifier and time-over threshold (TOT) discriminator. TOT discriminators work by only accepting input pulses which are above a predefined voltage threshold. Once the input pulse satisfies the threshold, the pulse is digitised to a logic pulse. The logic pulse width is determined by the magnitude of the analogue pulse. The larger the pulse, the wider its crossover with respect to the threshold. This can be used to measure the magnitude of the input pulse (see Figure 3.) The NINO was selected because of it very short dead-time and accuracy of determining the input charge with a jitter of 30ps on the leading edge signal. The total dead-time between events is about $3 \mathrm{~ns},{ }^{10}$ which is better than the MCP dead time. A 4-channel NINO corresponding to the 4 corner anodes of the CDIR anode was used. Varying pulse widths for each corner anode are used to determine the relative magnitude of the charge as it is capacitively spread to these anodes. If the NINO is stimulated with known charges, the corresponding pulse width can be measured in order to produce a lookup table which can be used to determine input charge from pulse width. This calibration is particularly useful for MCP imaging systems where the input electron cloud charge cannot be easily determined. This allows for centroiding of the incident charge cloud and acts as an implementation of position sensitivity for the device. 


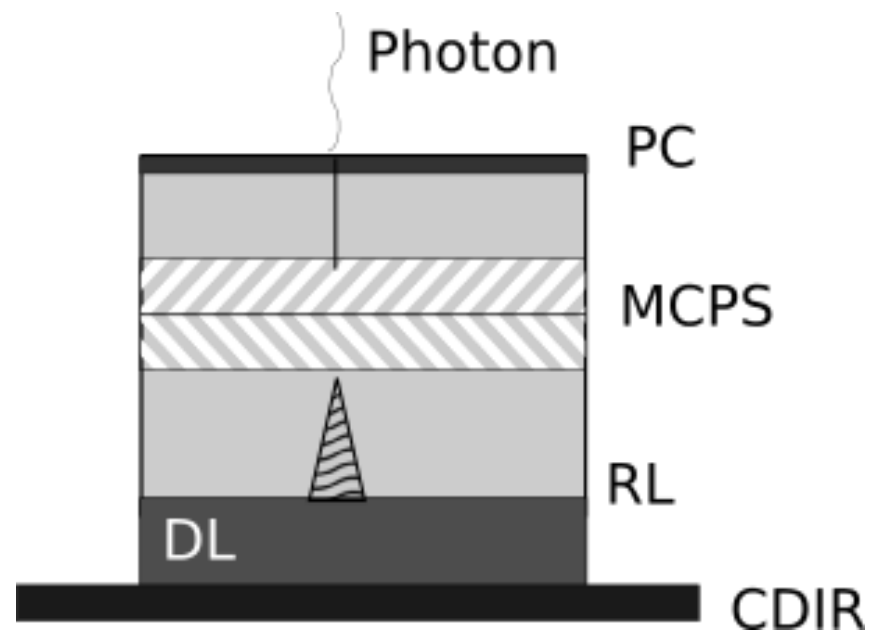

Figure 2. The detector system. PC: photo-cathode, MCPS MCP stack, RL resistive layer, DL dielectric layer.

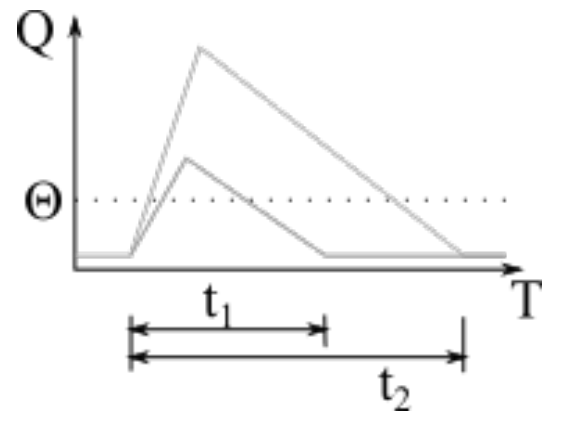

Figure 3. An example of how time over threshold, TOT, works. Q is the actual charge, T is time. $\Theta$ is some threshold above the background noise level. By knowing the charge and decay rates and measuring the times $t_{1}$ and $t_{2}$ the amplitude of the pulse can be calculated.

\subsection{HPTDC}

The HPTDC module consists of a TDC chip and a field programmable gate array (FPGA) which contains data processing routines. This device can be used in one of two triggering modes. Either the TDC can be used as a master trigger which can use a laser source as a slave, or it can be a slave to a laser source thus being triggered externally. Each of the four NINO channels is coupled to one of the HPTDC channels. The TDC chip digitizes the logic pulse of each NINO channel by recording the leading and trailing times of the pulse, relative to the TDC trigger. The FPGA packages this data in 32-bit words. The pulse width is determined by a simple subtraction of the lead-time from the trail-time. A pulse width vs. input charge lookup table for the NINO can be used to determine input charge for each channel and hence the position of the charge cloud.

\section{SPIM}

This section will now describe the microscope system built by the authors. The illumination was provided by a PicoQuant LDH-D-C-470 pulsed diode laser, with a wavelength of $\lambda=470 \pm 10 \mathrm{~nm}$, driven by a PicoQuant PDL 800-D single channel driver. This is capable of operating in both CW and pulsed mode with a pulse width of $<70 \mathrm{~ns}$ with a power of $0.3 \mathrm{~mW}$ for low power applications and a pulse width of $<300 \mathrm{~ns}$ for powers of $1 \mathrm{~W}$, It has a user selectable repetition rate of upto $40 \mathrm{MHz}$, but in our case the pulse was triggered from the HPTDC circuit. Although this may seem suitable for SPIM the beam profile produced by the laser is poor making a diffraction limited light sheet impossible to produce. Experimentally we could only achieve a light sheet of approximately $10 \mu m$. To improve on this we coupled the laser to a $9 \mu m$ core fibre optic. For this system this is still operating with multi-mode propagation but narrower fibre cores will result in too much power loss. 
After the fibre collimator, the beam is now circular and we convert this into a sheet using a telescope built of two cylindrical plano-convex lenses giving a magnification of $\times 3.4$ in one direction only. This fills the rear pupil of a 10X Mitutoyo Plan Apo Infinity-Corrected Long Working Distance Objective, in one direction ensuring the resulting is as diffraction limited as possible. To give a light sheet an additional $150 \mathrm{~mm}$ focal length plano-convex cylindrical lens is placed $150 \mathrm{~mm}$ in front of the objectives rear focal plane in the orthogonal direction to the first two lenses. This produces a collimated beam in one plane, and it is focused in the second plane, hence a light sheet.

The sample is then placed in a water filled sample chamber. The position can be controlled with a three axis stepper controlled stage and the sample can be rotated using an additional stepper motor.

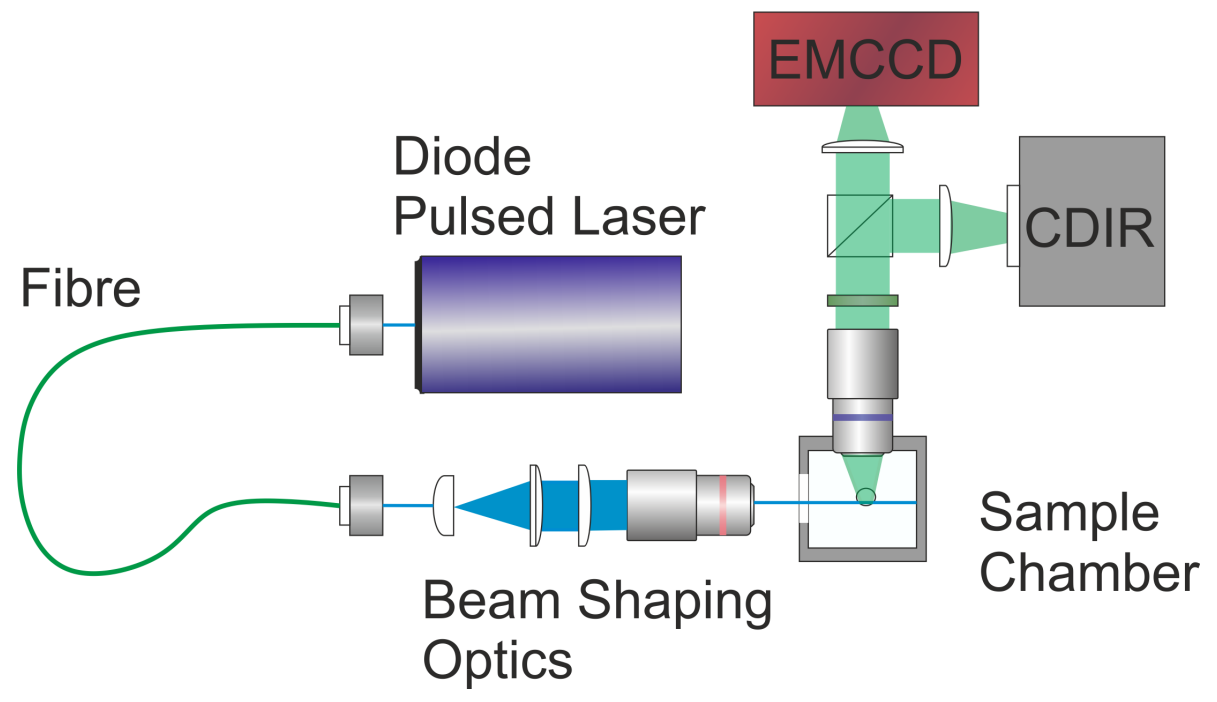

Figure 4. The SPIM optical layout.

An infinity color corrected water immersion objective (Plan-Apochromat, IR 20x/0.80 W (WD $=1.7 m m$ ), Zeiss) was used to collect and transfer the fluorescence into a collimated beam. A 488nm Yokogawa emission filter (FF495 EM01, Semrock) was placed behind the observation objective to filter out the excitation laser and only allow fluorescence at certain wavelengths $(\approx 497-560 \mathrm{~nm})$ to pass through.

A 10 : 90 reflection - transmission cubic beam splitter then reflects $10 \%$ of the light to the CDIR sensor, and 90\% towards a MP Vision EMCCD, provided by Lumitek. The EMCCD camera provides an intensity image of the subject, whilst the CDIR provides the lifetime information. Since the CDIR has active area of $25 \mathrm{~mm}$ diameter, which is larger than most cameras, an additional telescope was added to the CDIR optical arm.

\section{RESULTS}

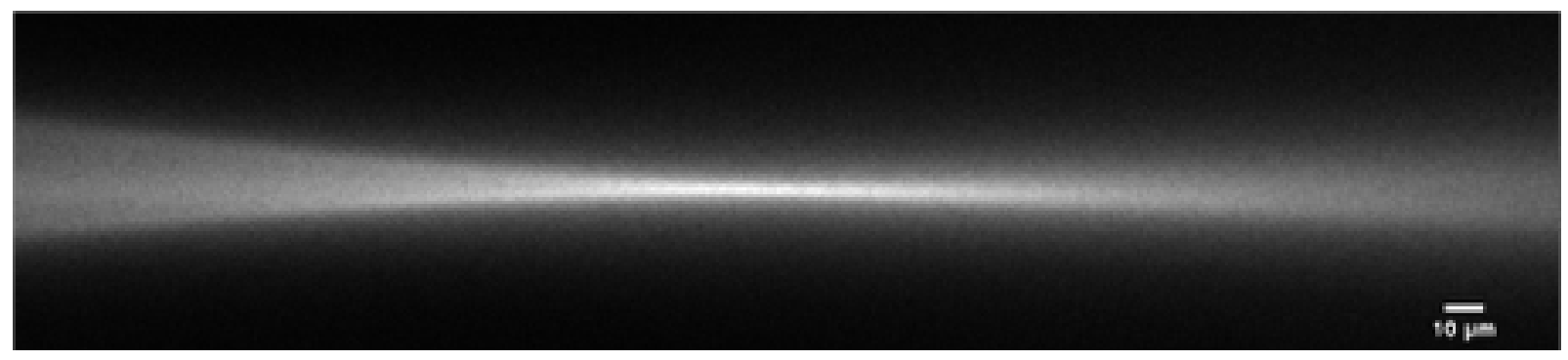

Figure 5. An image of the light sheet taken from above to measure its thickness. 
In order to measure the IRF, the emission filter, which was used to filter the excitation laser, was removed. A cover-slip was placed in the detection chamber and was turned $45^{\circ}$ to reflect the laser towards to detection objective. So the laser photon arrived on detector was counted according to time. The FWHM of the laser pulse was found at $411.38 \pm 14.75 \mathrm{ps}$ at $40 \%$ laser power.

To calibrate the spatial response of the CDIR, the filter was replaced and a fluorescent micro-bead was imaged. The position of the bead was moved in a $11 \times 11$ grid and the image captured on both the CDIR and the EMCCD. A composite image of a 121 images was then produced which is shown in Figure 6. It can be seen that the CDIR has a significant degree of distortion, especially at the edge of the image. To compensate for this, a polynomial was fitted to the images and this was used to correct for $\mathrm{x}-\mathrm{y}$ photon position data.
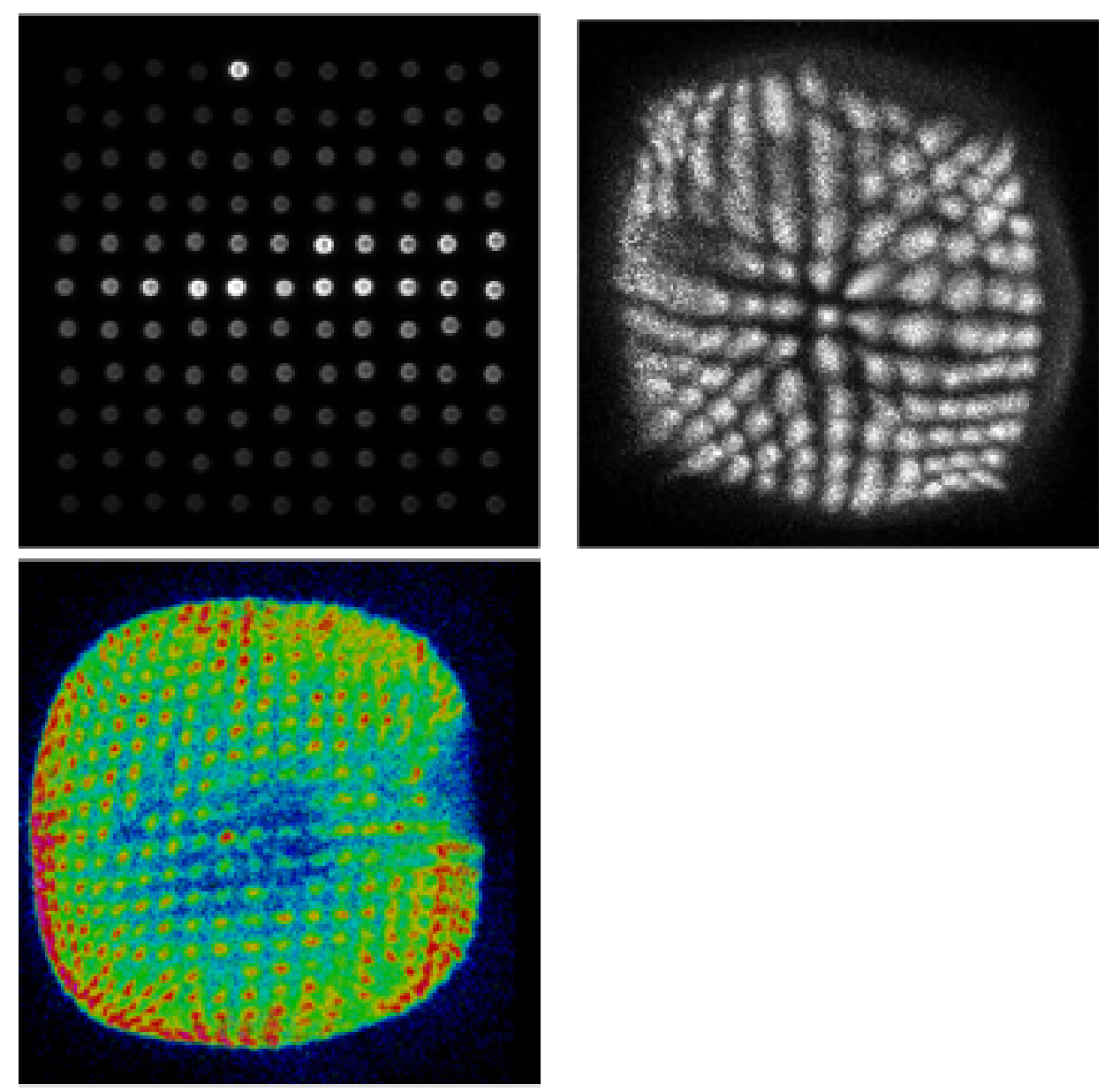

Figure 6. CDIR distorted images. Top left: The EMCCD composite image of the beads; Top-Right: The CDIR composite image; Bottom-Left: The corrected CDIR image.

Although the CDIR camera suffered severe distortion around the edges, we were able to prove that this distortion does not affect the spatial uniformity of the measured fluorescence lifetime. We measured the life time of Oregon Green Bapta-1 (OGB-1) in a series of 11 buffers of varying free $\mathrm{Ca}^{2+}$. Figure 7 shows the lifetime images of two of the buffers, showing the results have a reasonable uniformity.

Figure 8 shows an epifluorescence (green) and bright field (white) composite image of tissue dissected from a Drosophila larvae that was incubated in OGB-1 AM media. Fat body cell and salivary gland cells are indicated on the image. Two of the regions were also imaged using the CDIR/EMCCD and two exponentials fitted to the data. These are shown in figure 9. Although the fluorescence intensity images show a higher intensity in fat body these results show that it is in fact the salivary glands that have a higher concentration of $\mathrm{Ca}^{2+}$. 

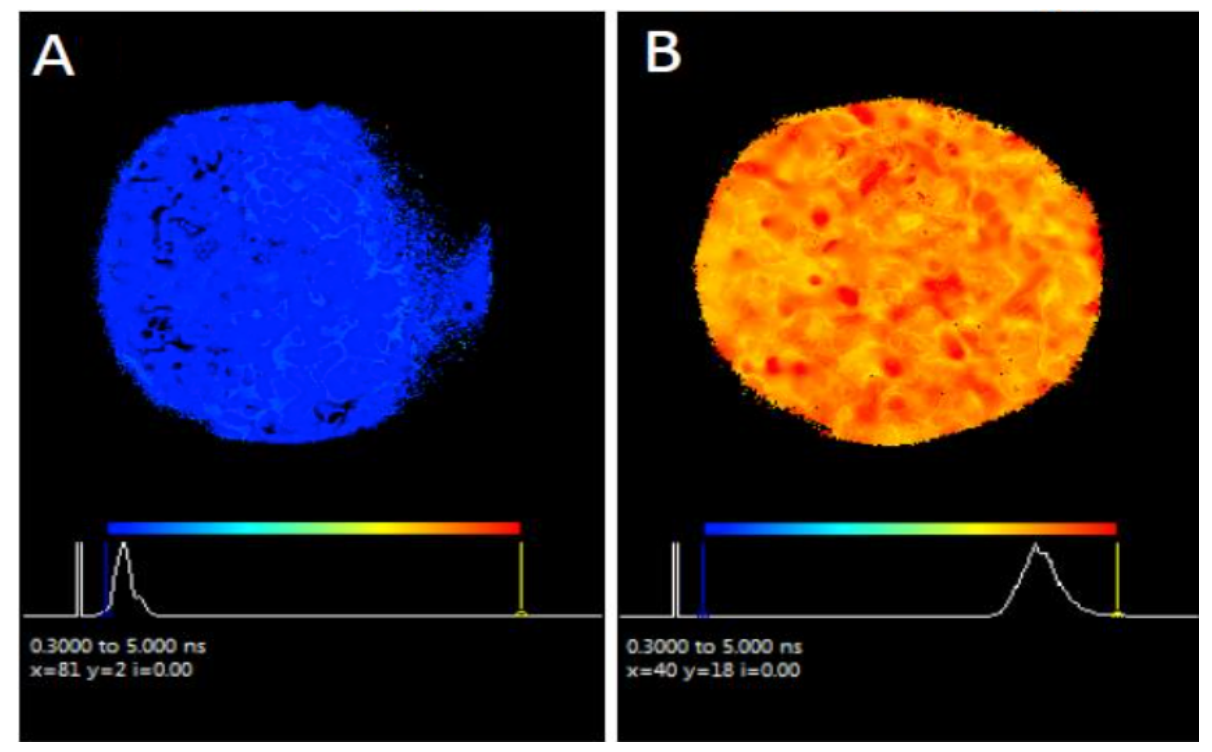

Figure 7. CDIR lifetime images of two buffers with A: $0 m M$ of $\mathrm{Ca}^{2+}$ and B: $39 \mu M$ of $\mathrm{Ca}^{2+}$. The images where produced by fitting a single exponential curve to the data.

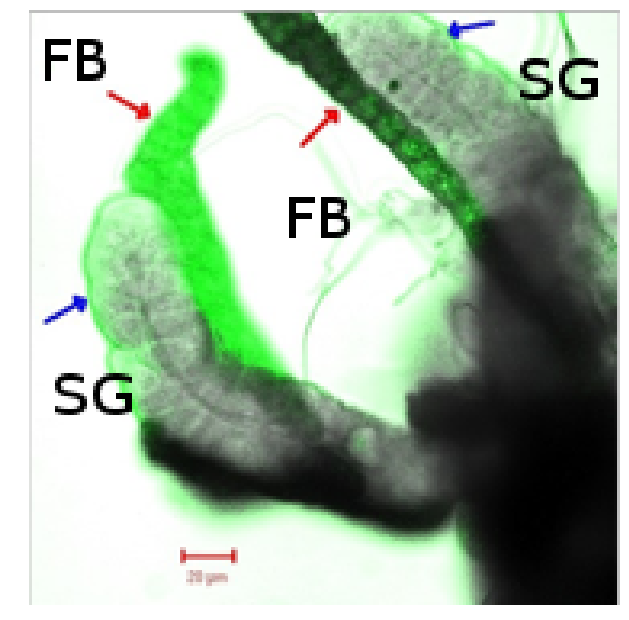

Figure 8. Drosophila larvae. FB indicates fat body cells, SG indicates salivary gland cells.

\section{CONCLUSIONS}

In this paper we have presented a new microscope system designed around a Photek CDIR MCP detector. We have described how this novel detector works and demonstrated it. The microscope is a custom designed SPIM using a pulsed laser diode as the source. We have shown that the temporal response is uniform across the device and the measurements so good correlation between them and the theoretical results. The detector does exhibit a degree of distortion, however, the distortion is purely in the $\mathrm{x}$-y direction and does not effect the temporal resolution characteristics of the device. The distortion can be partially corrected by using a polynomial interpolation to correct the $\mathrm{x}-\mathrm{y}$ positions of the photon.

\section{ACKNOWLEDGMENTS}

The author would like thank Tom Coneely and the staff at Photek Ltd ${ }^{8}$ and Lumintek for developing the CDIR system and their support and work to produce this paper. 


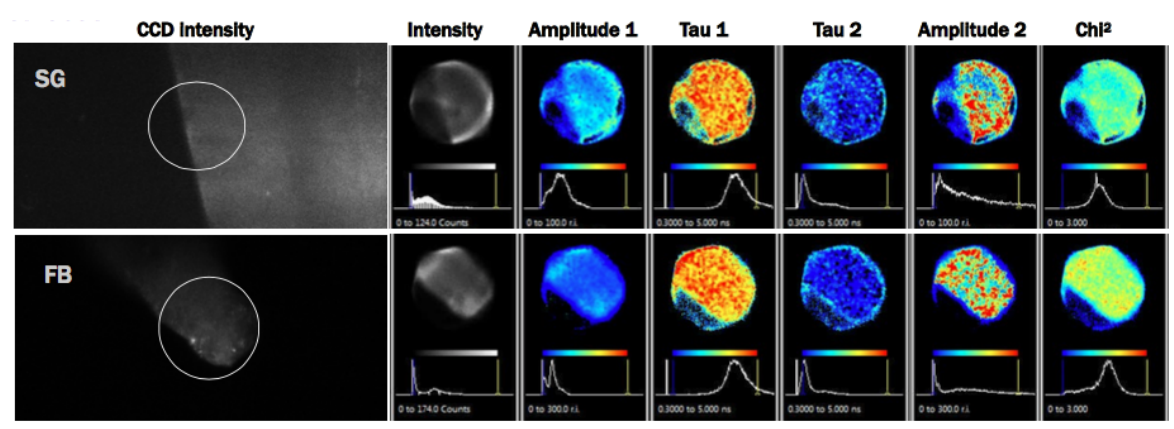

Figure 9. Drosophila larvae imaged using the CDIR with data fitting. FB indicates fat body cells, SG indicates salivary gland cells.

\section{REFERENCES}

[1] Lakowicz, J., [Principles of Fluorescence Spectroscopy], Springer, New York (2010).

[2] T. Frach, G. Prescher, C. D. and Zwaans, B., "The digital silicon photomultiplier -system architecture and performance evaluation," Nuclear Science Symposium Conference (2010).

[3] Hoebe, R. A., Oven, C. H. V., Jr, T. W. J. G., Dhonukshe, P. B., Noorden, C. J. F. V., and Manders, E. M. M., "Controlled light-exposure microscopy reduces photobleaching and phototoxicity in fluorescence live-cell imaging," Nature Biotechnology 2, 249-253 (2007).

[4] Hoover, E. E. and Squier, J. A., "Advances in multiphoton microscopy technology," Nature Photonics 7, 93-101 (2013).

[5] Huisken, J., Swoger, J., Del Bene, F., Wittbrodt, J., and Stelzer, E. H. K., "Optical sectioning deep inside live embryos by selective plane illumination microscopy.," Science 305, 1007-1009 (Aug. 2004).

[6] Coyler, R. A., Siegmund, O. H. W., Tremsin, A. S., J. V. Vallerga, S. W., and Michalet, X., "Phasor imaging with a widefield photon-counting detector," Journal of Biomedical Optics 17, 016008-1 - 016008-12 (2012).

[7] Michalet, X., Seigmund, O. H. W., Vallerga, J. V., P. Jelinsky, J. E. M., and Weiss, S., "Photon- counting h33d detector for biological fluorescence," Nuclear Instruments and Methods in Physics Research Section A 567, 133-136 (2006).

[8] Photek Ltd., 26 Castleham Road, St Leonards on Sea, East Sussex, TN38 9NS, United Kingdom. http: //www.photek.com/.

[9] Lapington, J. S., "High speed imaging using a capacitive division technique," Nuclear Instruments and Methods in Physics Research A 695, 410-414 (2012).

[10] Anghinolfi, F., Jarron, P., Martemiyanov, A. N., Usenko, E., Wenninger, H., Williams, M. C., and Zichichi, A., "Nino: an ultra-fast and low-power front-end amplifier/discriminator asic designed for the multigap resisitive plate chamber," Nuclear Intsruments and Methods in Physics Research 533, 183-187 (2004). 